\title{
An 80-year-old women presented with parotid gland swelling
}

\author{
Rawnak Jahan, Rajashish Chakrabortty, Shamim Ahmed and Mohammed Atiqur Rahman
}

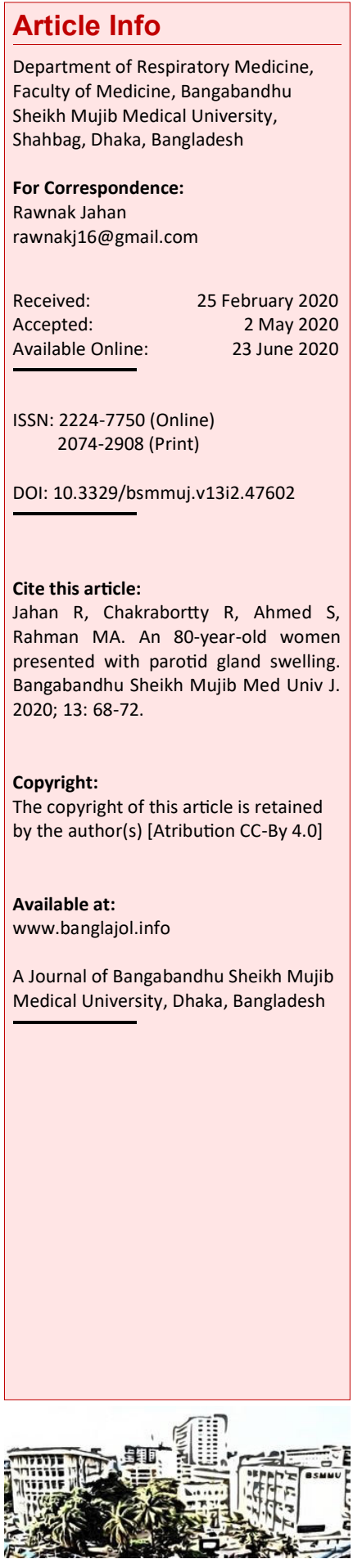

\section{Presentation of Case}

Dr. Rawnak Jahan (MD Resident): An 80-year-old woman hailing from Savar Upazilla, Dhaka got admitted into the Department on $7^{\text {th }}$ January 2020 with complains of swelling in the right side of cheek for the last 8 months (Figure 1A). Before that, the patient noticed a small nodular, painless swelling over anterior part of right ear, then it gradually increased in size and became hard but because of this swelling the patient did not feel any problem during eating or chewing foods. There was no history of fever, but the patient gave the history of occasional cough which was associated with scanty serous sputum. There were no history of hemoptysis, any shortness of breath, joint pain skin rash or dryness of mouth. The patient had anorexia and gave the history of weight loss evidenced by loosening her cloths. There was no history of previous tuberculosis or close contact with smear positive pulmonary tuberculosis. With this swelling she consulted several physicians. The fine needle aspiration cytology from the swelling was done twice but no conclusive diagnosis was made. The patient was hypertensive for the last 10 years and was on antihypertensive medication.

On general examination, she was afebrile, mildly anemic, and non-icteric. There was multiple palpable lymph nodes in the right cervical region, larger one was located in the right anterior chain which was about $2.5 \times 1.5$ $\mathrm{cm}$ in size, non-tender, firm, not fixed with overlying skin or any underlying structure. The discharging sinus was absent. Examination of the face revealed that there was a large swelling measuring about $8 \times 6 \mathrm{~cm}$ in the right cheek, anterior to the right ear, resemblance to parotid gland enlargement, which was hard in consistency, non-tender, fixed with overlying skin and underlying structures. There was slight erythematous skin. The local temperature was raised. Her blood pressure was $130 / 80 \mathrm{mmHg}$, pulse 80 beats/min and respiratory rate 18 breaths/min. All the systemic examination like respire-tory system, cardiovascular system, nervous system and abdomen revealed normal findings.

The patient was admitted with some investigations which showed hemoglobin was low (9.8 $\mathrm{g} / \mathrm{dL}$ ), erythrocyte sedimentation rate and total count were normal (Table I). The fine needle aspiration cytology from the right parotid swelling revealed chronic sialadenitis. The chest Xray posterolateral view showed bilateral miliary mottling (Figure 1B). The CT scan of chest showed bilateral miliary shadow and mediastinal lymphadenopathy (Figure 2).

So, based on history, physical examination and

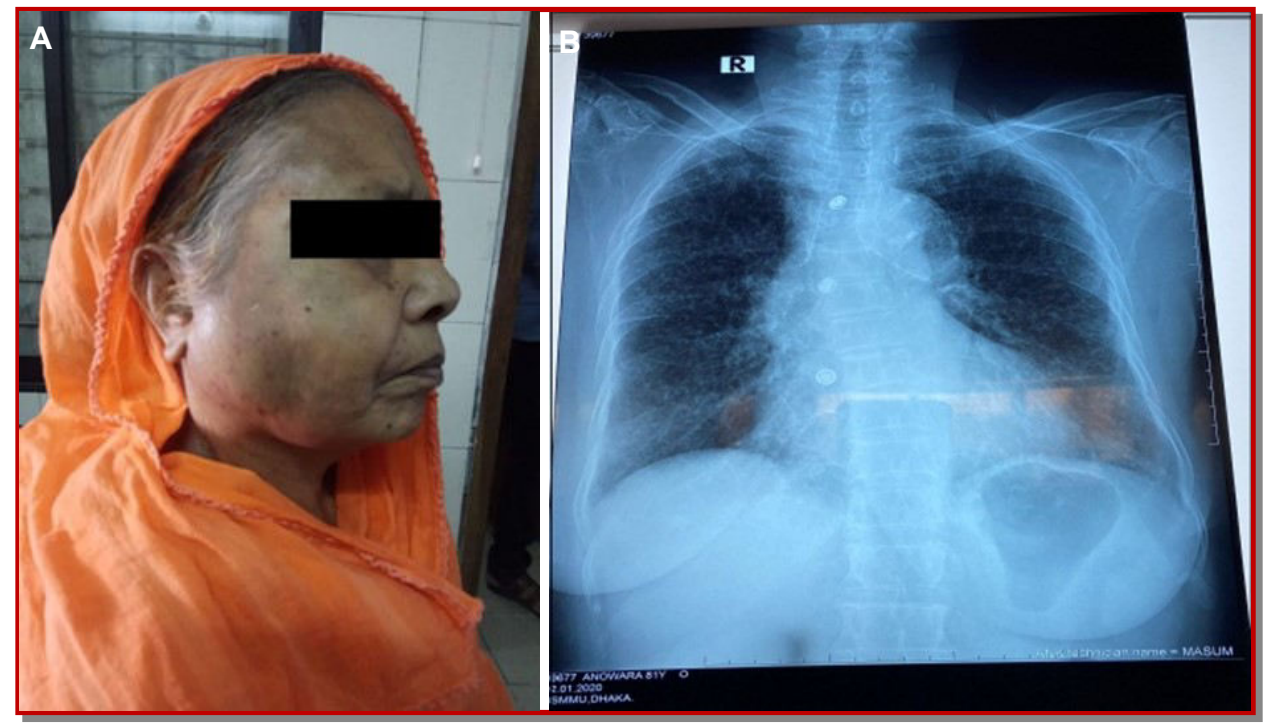

Figure 1: Patient with right parotid gland swelling (A); Chest X-ray posteroanterior view (B) 


\begin{tabular}{|lcr|}
\hline \multicolumn{3}{|c|}{ Table I } \\
Investigation profile \\
\hline Investigations & Findings & $\begin{array}{r}\text { Reference } \\
\text { value }\end{array}$ \\
\hline White cell count $\left(\times 10^{9} / \mathrm{L}\right)$ & 7.5 & $4.5-11$ \\
Platelet count $\left(\times 10^{9} / \mathrm{L}\right)$ & 250 & $150-450$ \\
Erythrocyte sedimentation rate (mm in st $^{\text {st }}$ hour) & 16 & $0-10$ \\
Hemoglobin $(\mathrm{g} / \mathrm{dL})$ & 9.8 & $13-17$ \\
Lactate dehydrogenase $(\mathrm{U} / \mathrm{L})$ & 175 & \\
Serum creatinine $(\mathrm{mg} / \mathrm{dL})$ & 1.0 & $0.4-0.7$ \\
Sputum for AFB stain & Not found & \\
Sputum for culture & No growth & \\
Mantoux test & Negative & \\
\hline
\end{tabular}

investigation findings we would like to draw a provisional diagnosis.

\section{Provisional Diagnosis \\ Right parotid gland tumor with lung metastases}

\section{Differential Diagnosis}

Dr. Rajashish Chakrabortty (Associate Professor): As the patient had miliary shadow in the chest imaging and lymph node enlargement was present, it could be a case of disseminated tuberculosis involving parotid gland, lymph node and lung.

Dr. Abir Hasan Dip (MD Resident): As the patient had parotid enlargement and diffuse chest

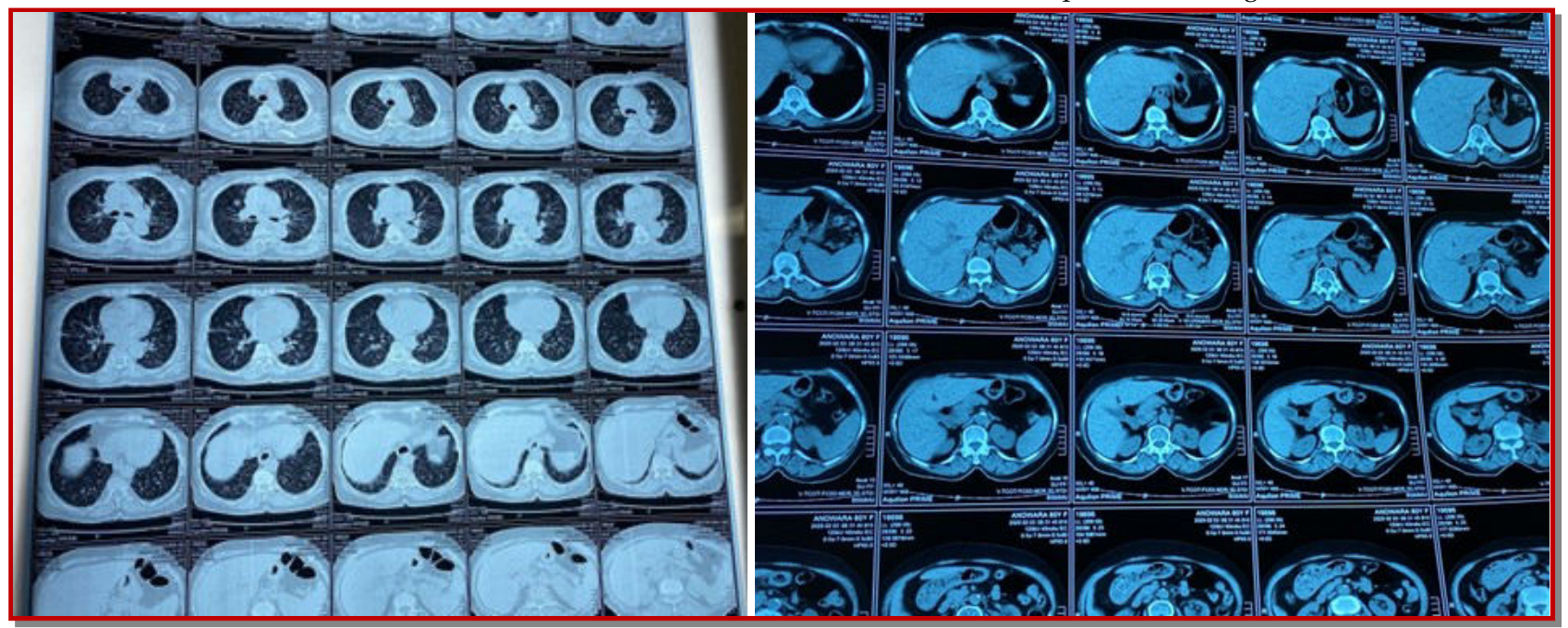

Figure 2: CT scan of the chest (A) and abdomen (B) with contrast

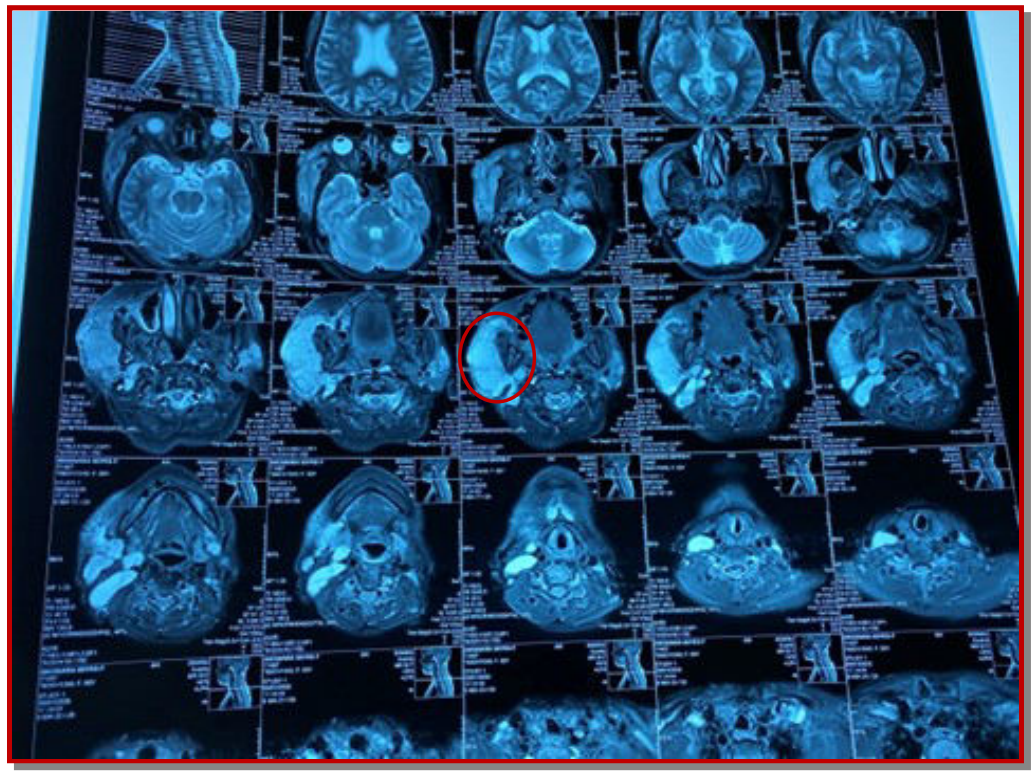

infiltration, sarcoidosis could be a differential diagnosis.

Dr. Shamim Ahmed (Associate Professor): Though it is vary rare but lymphoma should be considered as there are cervical and mediastinal lymphadenopathy.

\section{Parotid gland tumor with lung metastases}

Dr. Jahan: The tumor of salivary glands is uncommon and comprises only $0.3 \%$ of all cancers and $6 \%$ of all head and neck cancers. Among them, parotid tumors are the most common tumor, accounting 80 to $85 \%$ of all the salivary gland tumors. Most of the parotid tumors are benign but in $25 \%$ cases, it may be malignant. 1

Parotid tumors usually present as a lump or swelling. It is not possible to distinguish a benign tumor from a malignant one, but, fixation of the lump to the overlying skin, ulceration and induration of the mucosa, local lymph node enlargement and facial nerve palsy suggest malignancy.., 3

Figure 3: MRI of head and neck with contrast 
According to WHO parotid gland tumors can be classified into 5 categories: a) Benign epithelial tumor; b) malignant epithelial tumor- (mucoepidermoid carcinoma); c) soft tissue tumor (hemangioma); d) hematolymphoid tumor (Hodgkin's lymphoma), and e) secondary tumor. 1

Among them, mucoepidermoid carcinoma is the most common type. Distant metastases in the parotid cancer is rare but if occurs, then the most common site is the lungs. 4 The diagnosis of parotid tumor can be done by fine needle aspiration cytology. Imaging study may be helpful in staging and for surgical planning.

The points in favor of parotid malignancy in this patient are a) old age, b) painless lump, c) hard, fixed lump, and d) lymph node enlargement.

The points against are a) no facial nerve involvement, b) local temperature raised, c) miliary shadow in the lung, and d) FNAC report - chronic sialadenitis.

\section{Disseminated tuberculosis}

Dr. Chakrabortty: Disseminated tuberculosis is a contagious mycobacterial infection in which mycobacterias have spread from the lungs to other parts of the body through the blood or lymph system.

The usual site of tuberculosis is the lungs (pulmonary tuberculosis) but other organs can also be involved. The extra pulmonary tuberculosis accounts for approximately $20 \%$ of overall active tuberculosis and can be seen in the lymph nodes, meninges, bones and kidneys. $\underline{5}$

Tuberculous lymphadenitis is the most common form of extrapulmonary tuberculosis and the cervical lymph nodes are commonly involved. However, the parotid gland involvement is extremely rare, even in countries in which tuberculosis is endemic. This may be due to inhibitory effect of saliva on the mycobacteria. $\frac{5}{5}$ So, the diagnosis of parotid tuberculosis needs a high degree of clinical suspicion.

The pathogenesis of parotid tuberculosis remains unclear. Involvement of the parotid gland and lymph nodes may be developed in two ways: a) hematogenous or lymphatic spread from a distant primary lung focus; b) mycobacterial infection in the oral cavity liberates the mycobacterium that ascent into the salivary gland via its duct or pass to its associated lymph nodes via lymphatic drainage. 6

There are variable clinical presentations of parotid gland involvement with tuberculosis commonly presents as a localized mass. It may also present as an acute sialadenitis with diffuse glandular enlargement or it may present as periauricular fistula or as an abscess.
The diagnosis of parotid tuberculosis is difficult in the absence of clinical disease in the lung without any systemic manifestations. Most of the cases present with slow growing mass over 2 to 6 months and indistinguishable from the parotid neoplasm. Physical examination in general is unrewarding. A chest radiograph may be helpful in case of associated pulmonary tuberculosis. 7

The definitive diagnosis can be made by the detection of mycobacterium from diagnostic specimen. The fine needle aspiration cytology from the parotid gland is highly sensitive $(81-100 \%)$ and specific $(94-100 \%)$. Other diagnostic procedure such as MRI is helpful to delineate the nature of the disease. Incisional biopsy or drainage should not be used as there is a chance of cutaneous fistula.

In this case, as the patient had long standing history of slow growing mass, cervical lymphadenopathy and miliary shadow in imaging, so the tuberculosis should be excluded.

\section{Sarcoisosis}

Dr. Dip: Sarcoidosis is a multisystem granulometous disorder of unknown etiology that is characterized by the presence of non-caseating granulomas. Over $90 \%$ of cases, it effects the lungs but the condition can involve almost any organ. The pulmonary involvement may be present with insidious manner with cough, exertional breathlessness and radiographic infiltrates. The chest findings are often unremarkable. Other common presentations are erythema nodosum, arthralgia, occular symptoms, skin sarcoid, superficial lymphadenopathy, cranial nerve palsies, hypercalcaemia, nephrocalcinosis, parotid enlargement, etc. $\underline{7}$

The parotid gland involvement is rare in sarcoidosis and occurs in about $6 \%$ of cases. 8 The parotid sarcoidosis is presented as a persistant asymptomatic or painful, diffuse, non-nodular parotid swelling. 9 It is more often bilateral and commonly affects the female in $3^{\text {rd }}$ or $4^{\text {th }}$ decades. There is a rare manifestation of sarcoidosis known as Heerfordt syndrome which is characterized by the presence of parotid gland enlargement, facial palsy, anterior uveitis and fever. $\underline{10}$

The definitive diagnosis of parotid sarcoidosis is based on the patient's medical history, clinical examination and presence of non-caseating granuloma in the biopsy specimen. 11

In this case, though the patient had unilateral parotid swelling and the patient age did not suggestive of sarcoidosis but as there is diffuse pulmonary infiltrates and history of cough, weight loss, sarcoidosis can be considered though it is less likely.

Dr. Rawnak: To reach the correct diagnosis and to exclude the differentials, we have done further 
investigations. The MRI of head and neck with contrast was done which showed right parotid tumor with cervical lymphadenopathy (Figure 3). Repeat fine needle aspiration cytology from the swelling was done which showed Non-Hodgkin's lymphoma. The biopsy from the right cervical lymph node was done which showed nonHodgkin's lymphoma. To exclude the differentials and as the patient's chest radiology showed miliary shadow, we did sputum examination for acid-fast bacilli test, and the bronchoscopy followed by bronchoalveolar lavage study for acid-fast bacilli test. The Genexpert and malignancy were negative.

Finally we got clue that it was a rare case of nonHodgkin's lymphoma of the parotid gland with pulmonary infiltrates. For further confirmation, immunohistochemistry was advised.

\section{Dr. Shamim's Diagnosis}

Non-Hodgkin's lymphoma of parotid gland with lung infiltrates

\section{Discussion}

Dr. Ahmed: Lymphoma constitutes a neoplastic proliferation group arising from lymphocyte. Lymphomas can be categorized into two major subtypes, Hodgkin's lymphoma and non-Hodgkin's lymphoma. The Hodgkin's lymphoma appears as a node type disease including cervical, axillary and inguinal whereas non-Hodgkin's lymphoma localizes extranodally in the digestive tract, salivary gland and rarely the jaw. The non-Hodgkin's lymphoma has the most prevalence of all the lymphoma in head and neck accounting for $75 \%$ of all cases, but among tumor of parotid, the prevalence of lymphoma is rare, representing 1 to $4 \%$ of cases. 12

The malignant lymphomas of the parotid gland are often clinically unsuspected manifesting as nonspecific mass indistinguishable from other more common epithelial tumors. They are uncommon in patient younger than 50 years. The peak age of occurrence is in between 50 to 80 years. Most patients with lymphoma of parotid gland show a progressively enlarging, unilateral painless mass associated with enlarged cervical lymph nodes. Other unusual signs and symptoms that have been described are pain, facial nerve paresis, fixation of the mass to the overlying skin and deep tissue. $\underline{\underline{13}}$

The pulmonary involvement of non-Hodgkin's lymphoma can be classified as nodular type, bronchovascular lymphangitic type, pneumonic alveolar type and miliary hematogenous type. The most common radiologic presentation of nonHodgkin's lymphoma is a bronchovascular lymphangitic pattern. The miliary hematogenous infiltrates are uncommon and have described often in the end stage of the disease. This type of pulmonary infiltrate might implicate the rapid spreading of lymphoma cells via lymphatic and vascular pathways. $\underline{14}$

The diagnosis of lymphoma of the parotid gland is made by fine needle aspiration cytology especially combined with immunophenotyping and flow cytometry.

In case of this patient, she was 80 year old, had cervical and mediastinal lymphadenopathy and miliary shadow in the chest X-ray. The fine needle aspiration cytology from the parotid gland and the biopsy of the cervical lymphnode showed nonHodgkin's lymphoma. Immunohistochemistry showed CD20 positive and the findings are compatible with the nodal marginal zone B cell lymphoma. So, we diagnosed the patient as a case of non-Hodgkin's lymphoma. We consulted with the Department of Oncology for further management of the patient.

Dr. Samia Rahman (Resident): Is there any role of incisional biopsy for the diagnosis?

Dr. Rawnak: Incisional biopsy should be avoided even when fine needle aspiration cytology is unsuccessful in obtaining a diagnosis. An incisional biopsy should not be performed as the procedure has high rate of local recurrence and risk of facial nerve injury.

Dr. Rafsana (Resident): What is the further management plan?

Dr. Rawnak: We consulted the Department of Oncology and referred the patient in their department.

Dr. Abu Rayhan (Resident): What is the treatment option for the non-Hodgkin's lymphoma of parotid gland?

Dr. Rawnak: a) Chemotherapy in case of diffuse high grade lymphomas. It consists of rituximab- $\mathrm{CHOP}$ therapy (cyclophosphamide, hydroxydaunorubicin, vincristine, prednisolone); b) radiotherapy in case of localized low grade lymphomas; c) surgery paroditectomy (subtotal or total).

Dr. Ashique (Resident): What is the prognosis of this disease?

Dr. Rawnak: Malignant lymphoma originating in the parotid gland (intermediate or high grade) has satisfactory prognosis following radiotherapy and chemotherapy. 


\section{Final Diagnosis}

Non-Hodgkin's lymphoma with lung infiltrations

\section{Conflict of Interest}

Authors declare no conflict of interest.

\section{References}

1. Sowa P, Goroszkiewicz K, Szydelko J, Chechlinska J, Pluta K, Domka W, Misiolek M, Scierski W. A review of selected factors of salivary gland tumour formation and malignant transformation. Biomed Res Int. 2018; 2018.

2. Stodulski D, Mikaszewski B, Stankiewicz C. Signs and symptoms of parotid gland carcinoma and their prognostic value. Int J Oral Maxillofac Surg. 2012; 41: 801-06.

3. Mehanna $\mathrm{H}$, McQueen A, Robinson M, Paleri V. Salivary gland swellings. BMJ. 2012; 345; 36-41.

4. Ali S, Bryant R, Palmer FL, DiLorenzo M, Shah JP, Patel SG, Ganly I. Distant metastases in patients with carcinoma of the major salivary glands. Ann Surg Oncol. 2015; 22: 4014-19.

5. Gupta V, Patankar K, Shinde A, Bhosale C, Tamhane A. Tuberculosis of the parotid gland. Case Rep Radiol. 2012; 2012.
6. Birkent H, Karahatay S, Akcam T, Durmaz A, Ongoru O. Primary parotid tuberculosis mimicking parotid neoplasm: A case report. J Med Case Reports. 2008; 2: 1-4.

7. Reid PT, Innes JA. Sarcoidosis. In: Davidson's Principles and practice of medicine: A textbook for students and doctors. 22nd ed. London, Churchill Livingstone, 2014, pp 709-10.

8. James DG, Sharma OP. Parotid gland sarcoidosis. Sarcoidosis Vas Dif. 2000; 17: 27-32.

9. Iko BO, Chinwuba CE, Myers EM, Teal JS. Sarcoidosis of the parotid gland. BJR. 1986; 59: 547-52.

10. Fujiwara K, Furuta Y, Fukuda S. Two cases of Heerfordt's syndrome: A rare manifestation of sarcoidosis. Case Rep Otolaryngol. 2016; 2016.

11. Surattanont F, Mandel L, Wolinsky B. Bilateral parotid swelling caused by sarcoidosis. J Am Dent Assoc. 2002; 133: 738-41.

12. Lee HG, Lee JY, Song JM. Malignant lymphoma on parotid gland: A clinical case. J Korean Assoc Oral Maxillofac Surg. 2017; 43: 138-43.

13. Barnes L, Myers EN, Prokopakis EP. Primary malignant lymphoma of the parotid gland. Arch Otolaryngol Head Neck Surg. 1998; 124: 573-77.

14. Miyake S, Yoshizawa Y, Ohkouchi Y, Kurashima A, Hebisawa A. Non-Hodgkin's lymphoma with pulmonary infiltrates mimicking miliary tuberculosis. Intern Med. 1997; 36: 420-23. 